\title{
Frequency detuning effects for parametrically and directly excited elastic structures
}

\author{
Neumeyer, Stefan; van Gastel, M. H. M. ; Sorokin, Vladislav; Thomsen, Jon Juel
}

Published in:

Proceedings of the 5th ECCOMAS Thematic Conference on Computational Methods in Structural Dynamics and Earthquake Engineering

Publication date:

2015

Document Version

Peer reviewed version

Link back to DTU Orbit

Citation (APA):

Neumeyer, S., van Gastel, M. H. M., Sorokin, V., \& Thomsen, J. J. (2015). Frequency detuning effects for parametrically and directly excited elastic structures. In M. Papadrakakis, V. Papadopoulos, \& V. Plevris (Eds.), Proceedings of the 5th ECCOMAS Thematic Conference on Computational Methods in Structural Dynamics and Earthquake Engineering (Vol. 1, pp. 3029-3035). European Community on Computational Methods in Applied Sciences.

\section{General rights}

Copyright and moral rights for the publications made accessible in the public portal are retained by the authors and/or other copyright owners and it is a condition of accessing publications that users recognise and abide by the legal requirements associated with these rights.

- Users may download and print one copy of any publication from the public portal for the purpose of private study or research.

- You may not further distribute the material or use it for any profit-making activity or commercial gain

- You may freely distribute the URL identifying the publication in the public portal 


\title{
FREQUENCY DETUNING EFFECTS FOR PARAMETRICALLY AND DIRECTLY EXCITED ELASTIC STRUCTURES
}

\author{
S. Neumeyer ${ }^{1}$, M. H. M. van Gastel ${ }^{2}$, V. S. Sorokin ${ }^{1}$, and J. J. Thomsen ${ }^{1}$ \\ ${ }^{1}$ Department of Mechanical Engineering, Technical University of Denmark \\ Kgs. Lyngby, Denmark \\ stene@mek.dtu.dk \\ ${ }^{2}$ Department of Mechanical Engineering, Eindhoven University of Technology \\ Eindhoven, The Netherlands
}

Keywords: parametric and direct excitation, frequency detuning, nonlinear vibrations.

\begin{abstract}
This study investigates the frequency detuning effects of parametric and direct excitation for near-resonant nonlinear structural vibrations. Specifically, the detuning effects of a two-to-one frequency ratio between the parametric and direct excitation, and of a drift in natural frequency, are studied. These effects are investigated theoretically using a DuffingMathieu equation as the model system, and experimentally using a cantilever beam as the model object. The approximate analytical responses are derived using the method of varying amplitudes, and compared with results of direct numerical integration and experiments showing good agreement. For frequency detuned superthreshold parametric excitation some of the theoretical frequency-amplitude solution branches appear to merge. For some ranges of parametric excitation frequency a drop in experimental steady-state vibration amplitude was found, indicating performance degradation whereas for other frequency ranges, frequency detuning may yield an increased steady-state vibration amplitude. This makes frequency detuning a feature which can purposefully be avoided or utilized, dependent on the application.
\end{abstract}




\section{INTRODUCTION}

Structures subjected to combined parametric and direct excitation may experience frequency detuning between the parametric and direct excitation, e.g. caused by uncertainties with respect to physically adding the parametric component. Another cause of frequency detuning could be that the systems natural frequencies drift during operation due to, e.g. wear, damage, or varying mass loading. The effect of adding a superthreshold parametric excitation, where the response is dominated by the parametric component [1], to a directly excited system with perfect tuning, i.e. a two-to-one frequency ratio between the parametric and direct excitation, has recently been given some attention [2]. This was done because utilization of combined parametric and direct excitation may be useful for structural and mechanical vibrations [3, 4]. However frequency detuning has not been investigated for a system subjected to combined parametric and direct excitation, in particular not with superthreshold parametric excitation.

\section{MODEL SYSTEM}

As a model system relevant for elastic structures, a Duffing-Mathieu equation with independent parametric $\Omega_{p}$ and direct $\Omega_{d}$ excitation frequencies, is considered:

$$
\ddot{x}+\beta \dot{x}+\omega_{0}^{2}\left(1+p \cos \left(\Omega_{p} t\right)\right) x+\gamma x^{3}=d \cos \left(\Omega_{d} t+\phi\right),
$$

where $\left({ }^{\circ}\right)$ denotes temporal derivatives, $\beta=2 \omega_{0} \zeta$ where $\omega_{0}$ is the linear natural frequency and $\zeta$ is the damping ratio, $(\ldots) \omega_{0}^{2} x$ describes the linear elastic restoring force and $\gamma x^{3}$ describes the third-order nonlinear elastic restoring force, $p$ is a parametric excitation amplitude, $d$ is a direct excitation amplitude, $t$ is time, and $\phi$ is the phase between the parametric and direct excitation.

\section{THEORETICAL PREDICTIONS}

In applications involving imposed combined parametric and direct excitation, the frequencies $\Omega_{p}$ and $\Omega_{d}$ are typically tuned to be commensurate, e.g. with parametric amplifiers $\Omega_{p} / \Omega_{d}=2$, which provides a periodic steady-state response. Frequency detuning, by contrast, generally yields quasi-periodic steady-state responses. To solve (1) for the quasi-periodic response, we use the method of varying amplitudes (MVA) [5], and compare quantitatively with results of direct numerical integration and qualitatively with experimental observations. First a frequency detuning $\sigma_{p d}$ between the parametric and direct excitation is introduced, defined by:

$$
\Omega_{d}=\frac{1}{2} \Omega_{p}+\sigma_{p d}
$$

The MVA assumes a solution form consisting of a series of harmonics with time-varying amplitudes $X_{m 1}$ and $X_{m 2}$ :

$$
x(t)=\sum_{m=1}^{n} X_{m 1}(t) \cos \left(\frac{1}{2} m \Omega_{p} t\right)+X_{m 2}(t) \sin \left(\frac{1}{2} m \Omega_{p} t\right),
$$

which by contrast to perturbation methods such as multiple scales or averaging is without restrictions such as slowly varying amplitudes, or the presence of small parameters [5, 6]. Also, by contrast to the method of harmonic balance, where the coefficients $X_{m 1}$ and $X_{m 2}$ would be constants and (3) an approximation, the allowed time dependency of $X_{m 1}$ and $X_{m 2}$ means that (3) merely represents a shift of variables, which is exact for all values of $n$. The shift from the original dependent variable $x$ to $2 n$ new variables, $X_{m 1}$ and $X_{m 2}$, implies that $2 n$ equations are 
needed. This can be accomplished by introducing constraints in the form of $2 n-1$ additional equations. The constraints are introduced by substituting (3) into (1), and requiring $2 n-1$ groups of terms to equal zero. The $2 n^{\text {th }}$ equation includes all the remaining terms of the original equation, and the $2 n-1$ groups of terms are proposed to be the coefficients of the involved harmonic terms. Considering only the first two terms in (3), i.e. $n=1$, one obtains two equations for the amplitudes $X_{11}$ and $X_{12}$ :

$$
\begin{aligned}
\ddot{X}_{11}+\beta \dot{X}_{11}+\Omega_{p} \dot{X}_{12}+\frac{1}{2} \beta \Omega_{p} X_{12}+ & \left(\omega^{2}\left(1+\frac{1}{2} p\right)-\frac{1}{4} \Omega_{p}^{2}\right) X_{11} \\
& +\frac{3}{4} \gamma\left(X_{11}^{2}+X_{12}^{2}\right) X_{11}=d \cos \left(\sigma_{p d} t+\phi\right), \\
\ddot{X}_{12}+\beta \dot{X}_{12}-\Omega_{p} \dot{X}_{11}-\frac{1}{2} \beta \Omega_{p} X_{11}+ & \left(\omega^{2}\left(1-\frac{1}{2} p\right)-\frac{1}{4} \Omega_{p}^{2}\right) X_{12} \\
& +\frac{3}{4} \gamma\left(X_{11}^{2}+X_{12}^{2}\right) X_{12}=-d \sin \left(\sigma_{p d} t+\phi\right)+H .
\end{aligned}
$$

where $H$. denote higher harmonics. Until now approximations are not involved. Approximations are introduced by neglecting higher harmonics subsequently, which is adequate at small values of the nonlinearity term $\gamma x^{3}$ and parametric excitation term $p \omega_{0}^{2} x$, i.e. small compared to the linear stiffness term $\omega_{0}^{2} x$. Thus (1) is restated by (4) and (5), with $X_{11}$ and $X_{12}$ as the dependent variables instead of $x$. To solve (4) and (5), one employs the MVA again, ending up with four equations and four time dependent amplitudes, $Y_{111}, Y_{112}, Y_{121}$, and $Y_{122}$, to solve for. Solutions for these four amplitudes are lengthy and omitted here, but one ends up with a steady-state solution to (1):

$$
\begin{aligned}
x(t)= & \left(X_{110}+Y_{111} \cos \left(\sigma_{p d} t+\phi\right)+Y_{112} \sin \left(\sigma_{p d} t+\phi\right)\right) \cos \left(\frac{1}{2} \Omega_{p} t\right) \\
& +\left(X_{120}+Y_{121} \cos \left(\sigma_{p d} t+\phi\right)+Y_{122} \sin \left(\sigma_{p d} t+\phi\right)\right) \sin \left(\frac{1}{2} \Omega_{p} t\right) .
\end{aligned}
$$

Figs. 1(a,b) present, respectively, sub- and superthreshold steady-state vibration amplitudes, i.e. the maximum absolute values of (6), as a function of direct excitation frequency for the perfectly tuned case $\left(\sigma_{p d}=0\right)$. Fig. 11(a) shows that for subthreshold parametric excitation a response which qualitatively resembles a nonlinear hardening Duffing oscillator frequency response is obtained. Fig. 1 (b) shows that adding superthreshold parametric excitation to a directly excited system yields a bistable amplified steady-state peak. The solid line denote stable solution branches and the dashed line denote unstable solution branches. Each solution branch has an unbounded response maximum, contrary to the bounded subthreshold steady-state vibration amplitude. Good agreement between the theoretical predictions and results of direct numerical integration is noted. A distinct feature of the superthreshold parametric near-resonant steady-state vibration amplitude compared to the subthreshold parametric near-resonant steadystate vibration amplitude is, that for a certain frequency range it increases significantly. This might be useful for sensor applications because the transition from a small amplitude to a large amplitude becomes more pronounced, and it might also be useful for energy harvesters since more energy can be harvested.

Figs. 2(a,b) show two detuned $\left(\sigma_{p d} \neq 0\right)$ superthreshold steady-state vibration amplitudes with, respectively, a frequency ratio $\Omega_{p} / \Omega_{d}=2.05$ and 1.95 between the parametric and direct excitation. The somewhat larger deviation between the approximate analytical solution and results of direct numerical integration around the intersection between the lower and upper solution branches can be reduced by introducing higher harmonics, though omitted here since the calculations become more lengthy with only minor increase in accuracy. For some frequencies 

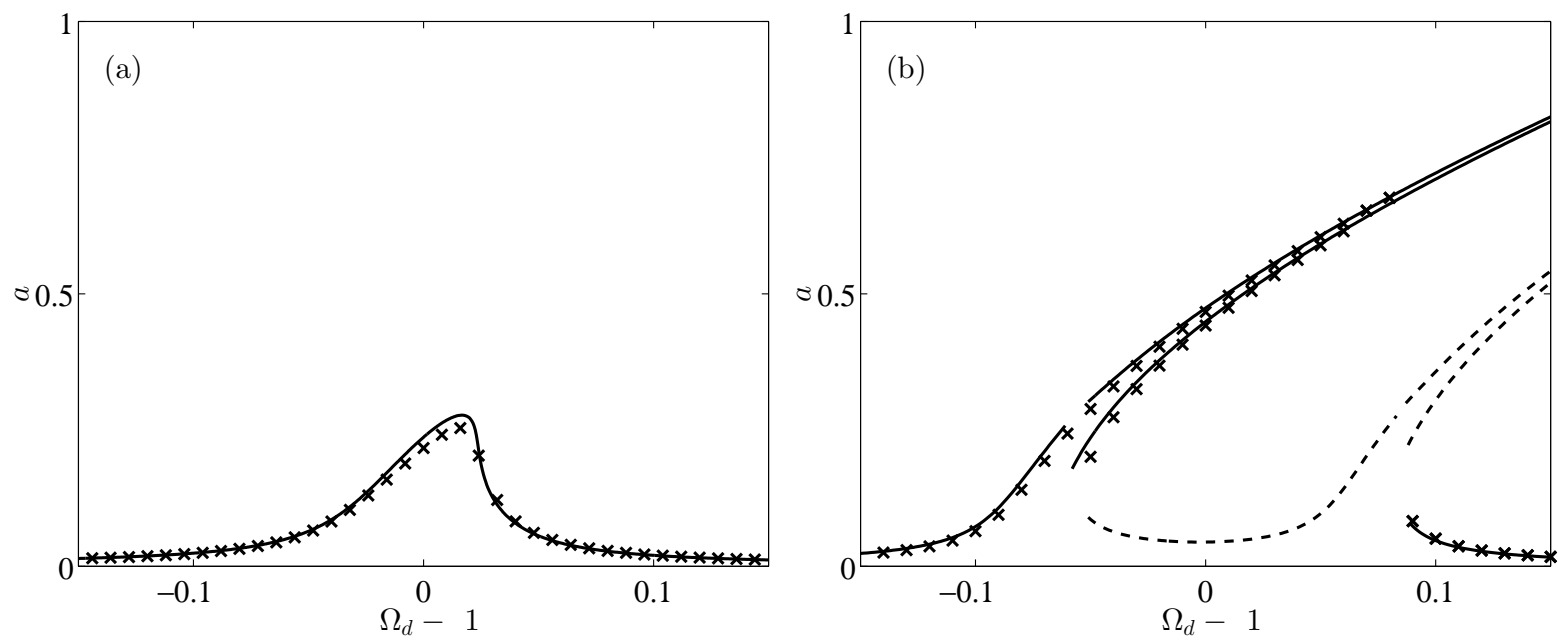

Figure 1: Theoretical steady-state vibration amplitude as a function of direct excitation frequency $\Omega-1$ for (a) subthreshold parametric excitation $(p=0.15)$, and $(b)$ superthreshold parametric excitation $(p=0.35)$. Solid and dashed lines denote, respectively, approximate analytical stable and unstable responses, symbols denote direct numerical integration. $\left(\gamma=0.1, d=0.01, \beta=0.1, \phi=-\pi / 4, \Omega_{p} / \Omega_{d}=2, \sigma_{p d}=0\right)$.

up to five solution branches coexist. Two of these are duplicates in the frequency-amplitude relation except for a difference in phase, i.e. with frequency detuning between the parametric and direct excitation, the bistable amplified peak of fig. 1(b) appears to merge into one line. Jumps are observed, not only during a downsweep, but also during an upsweep. The upsweep jump is, for the chosen parameters, considerably smaller than for the downsweep, but this might be otherwise for other parameter values. Also, the frequency distance between the two peaks changes as function of frequency detuning between the parametric and direct excitation. For a negative nonlinear coefficient $\gamma$ the steady-state vibration amplitude bends to the left instead of to the right, and the upper parts of the stable and unstable solution branches interchange stability.
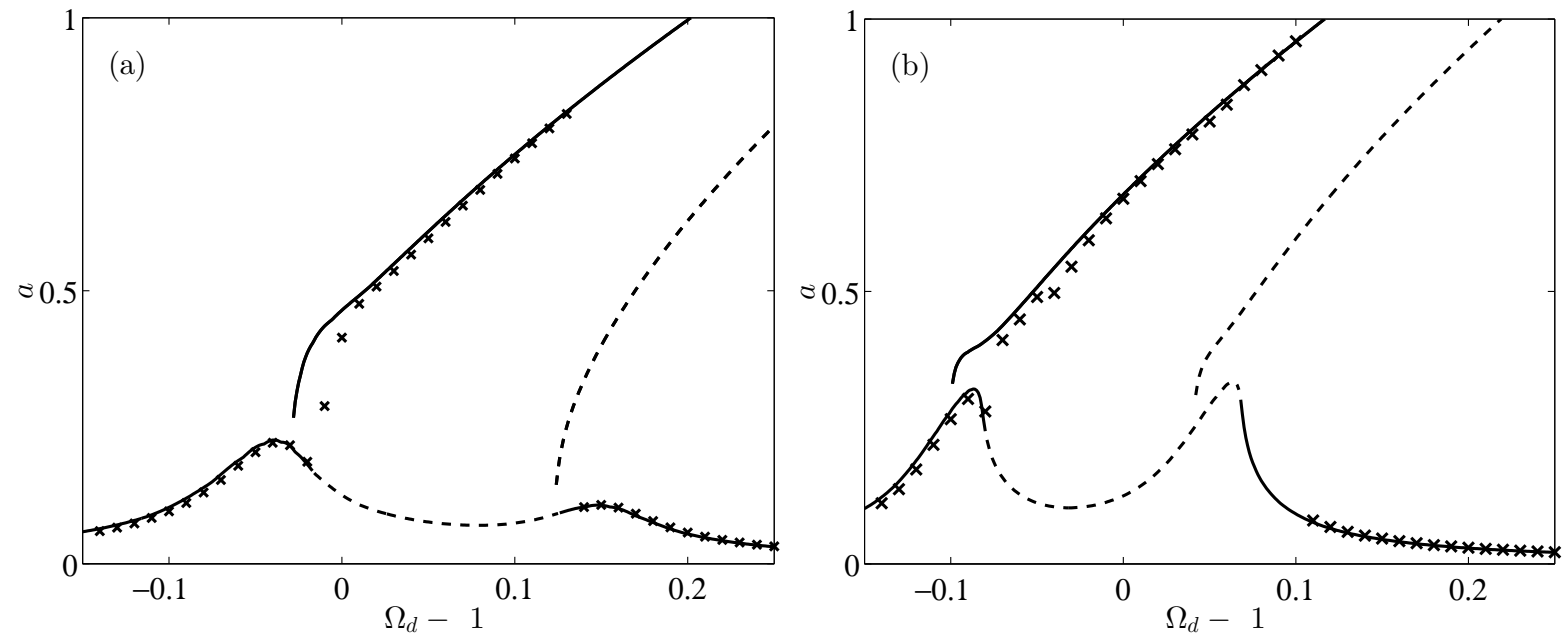

Figure 2: Theoretical superthreshold steady-state vibration amplitude as a function of direct excitation frequency $\Omega-1$ for (a) $\Omega_{p} / \Omega_{d}=2.05\left(\sigma_{p d}=-0.025\right)$, and (b) $\Omega_{p} / \Omega_{d}=1.95\left(\sigma_{p d}=0.025\right)$. $(p=0.35)$. 
Compared to the perfectly tuned case, confer fig. 1(b), an upsweep jump emerges, and both peaks appear to shift to either the left or right of zero direct excitation frequency. Furthermore, superthreshold parametric excitation increases the near-resonant steady-state vibration amplitude as compared to employing subthreshold parametric excitation, and a slight frequency detuning of superthreshold parametric excitation can result in a further increase in steady-state vibration amplitude.

The theoretical part showed good agreement between approximate analytical responses and results of direct numerical integration, both in the perfectly tuned and detuned cases. For detuned superthreshold parametric excitation some of the frequency-amplitude solution branches appear to merge, and the frequency distance between the two peaks changes.

\section{EXPERIMENTAL OBSERVATIONS}

Fig. 3 shows the experimental setup. A cantilever beam is fixed in a shaker with an angle between the imposed base-excitation and the axial direction of the cantilever beam, such that the transverse displacement of the cantilever beams free end is affected by both the parametric and direct excitation. The dual frequency $\left(\Omega_{d}, \Omega_{p}\right)$ signal to the shaker is produced by a waveform generator and amplified by a power amplifier. The transverse deflection of the cantilever beam is measured by a laser displacement sensor at the beam end and normalized with the beam length. For each excitation frequency detuning experiment both up- and downsweeps are conducted for the parametric and direct excitation frequencies. The direct excitation frequency is altered in an inner sweep, and the parametric excitation frequency in an outer sweep.

Figs. 4(a,b) show results for variation in direct and subthreshold parametric excitation frequencies. Fig. 4(a) provides an isometric view and fig. 4(b) the corresponding top view. As appears the maximum steady-state vibration amplitude is mostly sensitive to direct excitation frequency detuning (variations in $\tilde{\Omega}_{d}$ ), and much less to parametric excitation frequency detuning (variations in $\widetilde{\Omega}_{p}$ ). One response notch, entangled by a red circle, is observed alongside jumps occurring due to a nonlinear hardening response. Slight variations in the parametric and direct excitation frequencies around the notch can result in a significantly lower response.

Figs. 4(c,d) show results for variation in direct and superthreshold parametric excitation frequencies. As compared to the case with subthreshold parametric excitation, another notch appears making the frequency range with a higher steady-state vibration amplitude significantly smaller, albeit the maximum steady-state vibration amplitude increases. These notches are also considerably larger in the case of superthreshold parametric excitation, as compared to the subthreshold case. With superthreshold parametric excitation the steady-state vibration amplitude increases for a part of the frequency range, but another part of the frequency range, i.e. the

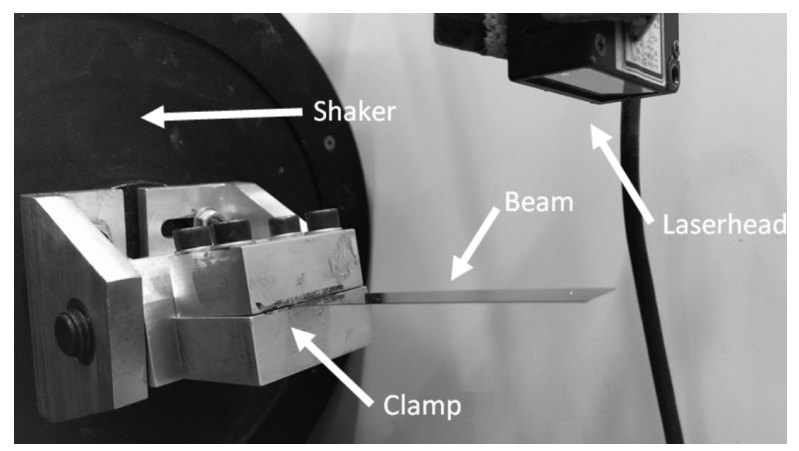

Figure 3: Experimental setup. 

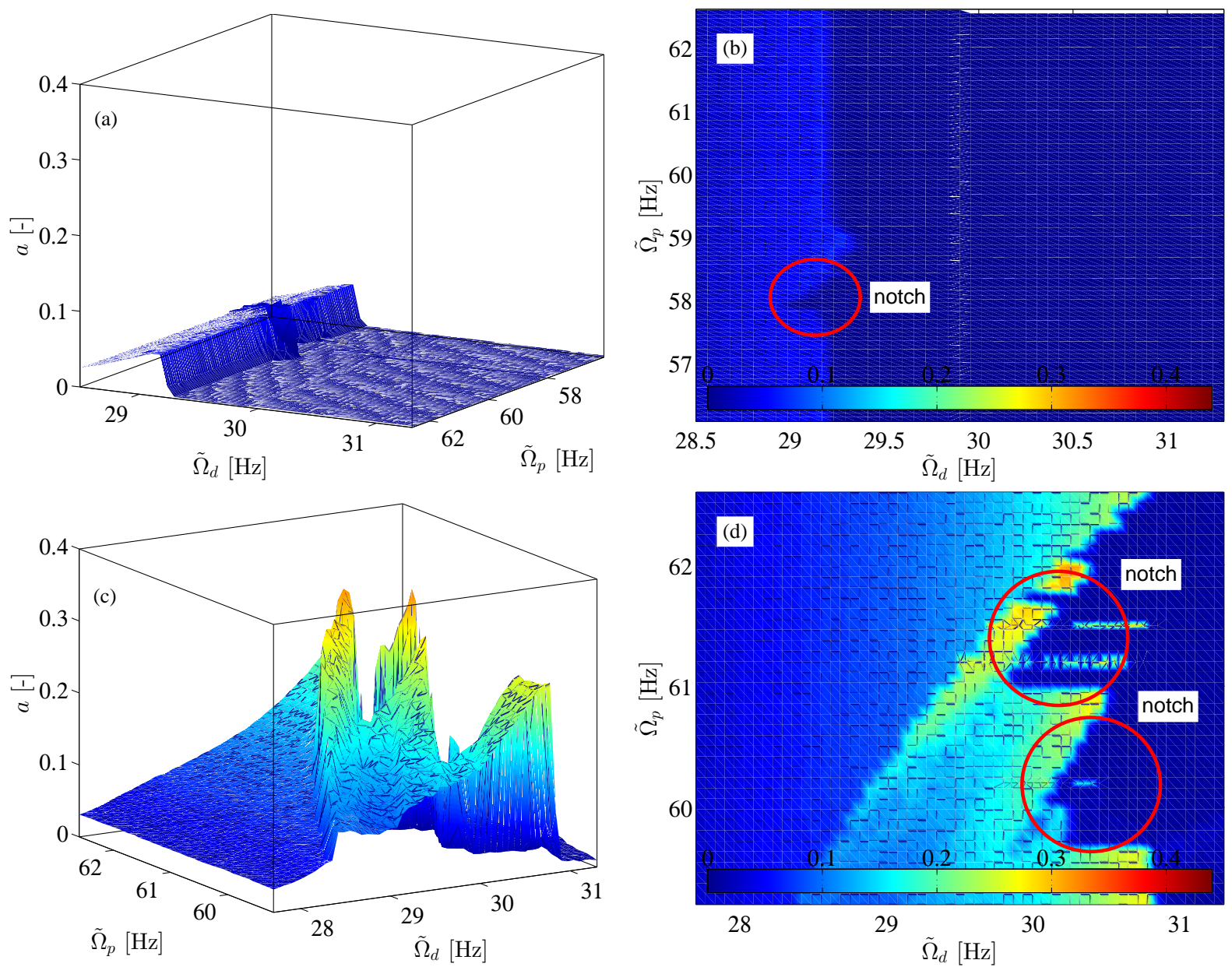

Figure 4: Experimental results: Isometric and top view of subthreshold (a,b) and superthreshold (c,d) steady-state vibration amplitude $a$ as a function of direct and parametric excitation frequencies using a downsweep with $\tilde{\Omega}_{d}$ fixed while $\tilde{\Omega}_{p}$ is varied.

area entangled by the extra notch, indicates that there exist additional frequency ranges where a smaller steady-state vibration amplitude is obtained. Outside the frequency region of the notches, detuning of both the direct and parametric excitation frequencies has a small influence on the response.

The theoretical predictions presented in figs. $2(a, b)$ depict two special cases of the experimental observations presented in figs. $4(\mathrm{c}, \mathrm{d})$; the two frequency ratios $\Omega_{p} / \Omega_{d}=2.05$ and 1.95 correspond to two distinct lines in figs. 4(c,d). Good agreement is noted: jumps and the overall qualitative behaviour appears similar, and the change in frequency distance between the two peaks as predicted theoretically, confer figs. 2(a,b), is also observed experimentally. Also, in both cases frequency detuning between parametric and direct excitation can increase the response. Thus, frequency detuning can result in both decreased and increased steady-state vibration amplitudes, making it an effect which should either be avoided or utilized dependent on the applications.

\section{CONCLUSIONS}

Frequency detuning effects for combined parametric and direct near-resonant excitations of nonlinear structural vibrations were investigated. This is relevant for applications where frequency detuning can be expected between the parametric and direct excitation, or between the 
parametric and direct excitation and the systems natural frequency during system operation. The studies were conducted theoretically using a Duffing-Mathieu equation as the model system, and experimentally using a cantilever beam as the model object. The approximate analytical responses were derived using the method of varying amplitudes, and compared with results of direct numerical integration, showing good agreement both in the perfectly tuned and detuned case. Good qualitative agreement also with experiments were noted. For detuned superthreshold parametric excitation some of the theoretical frequency-amplitude solution branches appear to merge. For some frequency detuning ranges a drop in experimental steady-state vibration amplitude was found, indicating performance degradation whereas for other frequency ranges, frequency detuning may yield an increased steady-state vibration amplitude. This makes frequency detuning a feature which can purposefully be avoided or utilized, dependent on the application, e.g. sensors and energy harvesters.

\section{REFERENCES}

[1] J.F. Rhoads, S.W. Shaw, The impact of nonlinearity on degenerate parametric amplifiers, Applied Physics Letters, 23, 234101.1-3, 2010.

[2] S. Neumeyer, J.J. Thomsen, Macroscale mechanical domain parametric amplification: superthreshold pumping and optimal excitation parameters, $1^{\text {st }}$ International Colloquium on Time-Periodic Systems: Current trends in theory and application (Euromech 532), Darmstadt, Germany, 2012.

[3] D. Rugar, P. Grütter, Mechanical parametric amplification and thermomechanical noise squeezing, Physical Review Letters, 67, 699-702, 1991

[4] M. Cartmell, Introduction to Linear, Parametric, and Nonlinear Vibrations, Chapman and Hall, 1990.

[5] V.S. Sorokin, J.J. Thomsen, Vibration suppression for strings with distributed loading using spatial cross-section modulation, Journal of Sound and Vibration, 335, 66-77, 2015

[6] A.H. Nayfeh, D.T. Mook, Nonlinear oscillations, Wiley, 1979. 